\title{
Survey of the Application Fields and Modeling Methods of Automotive Vehicle Dynamics Models
}

\author{
A. SzÁNTó1 ${ }^{1}$ S. HAJDU², K. DEÁK ${ }^{3}$ \\ ${ }^{1}$ The University of Debrecen, Faculty of Engineering, andras.szanto.0503@gmail.com \\ ${ }^{2}$ The University of Debrecen, Faculty of Engineering, hajdusandor@eng.unideb.hu \\ 3The University of Debrecen, Faculty of Engineering, deak.krisztian@eng.unideb.hu
}

\begin{abstract}
In this paper, a review is presented on automotive vehicle dynamics modeling. Applied vehicle dynamics models from various application fields are analyzed and classified in the first section. Vehicle dynamics models may be simplified because of different reasons: several control/estimation/analysis methods are suitable only for simplified models (e.g. using control-oriented models), or because of the computational cost. Detailed/truth models of vehicle dynamics represent another field of vehicle dynamics modeling, these models play an important role in the virtual prototyping of vehicles. In the second section, the main modeling considerations of vehicle dynamics are presented in longitudinal, lateral and vertical directions. Various physical effects must be considered in the case of vehicle dynamics modeling, a lot of these effects are significant only in a specific direction of the vehicle body, which is the main potential of model simplification. The section presents vehicle modeling considerations in all of the three translational directions of the vehicle body.
\end{abstract}

\section{Introduction}

In this paper, automotive vehicle dynamics modeling is reviewed from two main perspectives. In Section 1, we analyze and classify the applied vehicle models in various applications. There are different types of vehicle models utilized in practice, from simplified to detailed ones. A variety of arguments are shown why it is necessary to perform simplifications on the vehicle models. On the other hand, it is a fact that all methods are available to model vehicles in a realistic manner, these detailed models have potential in virtual prototype-based development in the vehicle industry. In Section 2, simplified vehicle models are analyzed in the longitudinal, lateral and vertical directions. The significant physical effects in all directions are pointed out. Combining the modeling considerations of each vehicle body directions could lead to a complete detailed/truth model, or the separate simplified models could be utilized in applications requiring simplified models.

\section{Vehicle dynamics models from the application perspective}

In this section, we present an overview of automotive vehicle dynamics models from the application perspective. Model-simplification and its reason are the basis of the classification. Vehicle dynamics models have a wide range of application fields, and these various fields assume different types of models. Vehicle dynamics must be considered in the field of estimation algorithms and control systems 
related to vehicles [1]-[10] [11]-[20], as well as in path planning algorithms [21]-[23]. The previously mentioned application fields are usually within the embedded systems, this is one of the reasons why these models are simplified versions of the so-called detailed/truth models. Another reason for using simplified models is that control/estimation and some analysis methods are usually applicable only to simplified or control-oriented models. Simplified vehicle models are used in powertrain simulations and optimizations [24], [25], where the main focus is on the powertrain modeling and detailed vehicle dynamics models are not required. There are various kinds of simplified vehicle models including at least one of the vehicle body directions - longitudinal, lateral or vertical translational directions, and roll, pitch or yaw rotational directions. The modeling considerations of vehicle models of these directions are presented in Section 2. Besides simplified models, there are detailed/truth vehicle models [26]-[29] that describe the real vehicle in sufficient detail, considering a six degrees of freedom vehicle body without constraints. These types of models are virtual prototypes of the vehicle, can evaluate estimation and control algorithms, and also can be used for high-fidelity validation of autonomous driving systems. There are other applications of vehicle models like crashworthiness models [30], and vibration models [31] analyzing the pothole-induced forces.

\subsection{Application of simplified vehicle models}

The main attribute of a simplified vehicle model is that instead of a six degrees of freedom vehicle body, one or more constraints are applied to it, which constraints can be translational and rotational. Applying constraints to a rigid body results in constraint equations - force or torque equilibrium equations. A model of this kind is generally described by a differential-algebraic system of equations (DAEs). Although in some cases a DAE system can be transformed into ordinary differential equations (ODEs), the most common way is to completely neglect the constrained directions (the constraint equations). In most control/estimation methods only ODEs can be handled, hence some transformation/neglection is necessary.

In all referenced sideslip estimations [4]-[7] vertical force components $\left(F_{z}\right)$ in the tire-road contact points are neglected, longitudinal and lateral forces do not depend on the vertical force components (e.g. $F_{x}(k a p p a)$ instead of $F_{x}\left(\right.$ kappa, $\left.F_{z}\right)$ is considered, where $F_{x}$ is the longitudinal force and kappa is the longitudinal slip). There are some longitudinal slip estimations [3] where $F_{x}$ (kappa, $F_{z}$ ) is considered.

\subsubsection{Simplified models for control/estimation/analysis applications}

In references [1]-[3] estimations are performed in the longitudinal direction. Typical estimations in the longitudinal direction consist of longitudinal slip and tire friction coefficient estimation [3], or mass and/or road grade (slope) estimation [1], [2]. Adaptive cruise control (ACC) is also significant only in the longitudinal direction [20], as well as anti-lock braking system (ABS).

Estimation and control are performed also in the lateral translational and yaw rotational directions [4]-[7], [20]. The main objectives in these directions are sideslip estimation [4], [6], [7], cross-wind estimation [20], and stability control [4], [5], [7]. Lane-keeping and path following control methods [12]-[14] are also highly dependent on the lateral and yaw dynamics of the vehicle. However, usually 
in these applications model-predictive control (MPC) is used, so even a detailed/truth model could be utilized (only the computational cost is a limitation).

In the vertical direction of the vehicle body, active suspension estimation and control algorithms [8], [9] are implemented. In these vertical models, the $F_{x}$ longitudinal and the $F_{y}$ lateral forces are usually neglected. Road profile estimations [17], [18] also utilize vertical vehicle models.

In the longitudinal and lateral directions (where $F_{x}$ and $F_{y}$ forces are considered), it is a question that what kind of tire model is utilized. There are constant tire models applied [5], [11], [19], [20] which can be interpreted as constant road conditions, the force-slip characteristics are constant. The estimation/control algorithms based on these tire models can be robust even is varying road conditions, but there is no estimated information about the road conditions. On the other hand, adaptive tire models can be utilized [3], [4], [6], the estimations based on these tire models provide real-time information about the road conditions. Usually, this kind of tire model is dependent on a scalar value that is in the estimated state vector, this results in the adaptive nature of it. The models used in some vehicle dynamics applications can completely neglect $F_{x}$ and $F_{y}$ tire models, because of two main reasons. One is that these forces are not significant in the specific application (like in the case of active suspension and road profile estimations). The other reason is that force estimation [6], [10] is performed directly instead of slip estimation, which means that the vehicle model used for estimation does not contain information about the tire characteristics, the forces are directly estimated (the estimated state vector contains force terms instead of slip terms). Because of the fact that the estimated force states are not dependent on tire models, this method can be interpreted as a measurement of the tire force-slip characteristics, tire models can be identified based on this kind of estimation [10]. There is another aspect of tire modeling. Tires have transient behavior which means that the forces do not develop instantly, but build as the tire rolls (the force-slip characteristics are only valid for steady-state). This property of tires is called the relaxation length [32], [33]. This property also can be considered in the estimation models of vehicles [11] (although the detailed/truth models of this phenomenon can be different than the models utilized for estimation).

Vehicle dynamics can be considered in the case of vehicle positioning systems. Sensors of microelectro-mechanical systems (MEMS) and inertial measurement units (IMU) are used in all fields of estimations related to vehicles, alongside with the signals available on the Controller Area Network (CAN bus). Global Positioning System (GPS) and compass sensors (or magnetometers) can provide absolute position and rotation information for vehicles, the combined usage of sensor fusion techniques and vehicle dynamics-based estimations is another research field [19].

There are analysis techniques which can provide analytic solutions only based on simplified vehicle models. These analytic (or symbolic) solutions can provide more insight into the essential connections between variables in a model, than the solutions of numerical analysis. A great example of this field is the analysis of pothole-induced contact forces [31].

\subsubsection{Simplification because of computational cost and other reasons}

Path planning is another application field of vehicle dynamics [21]-[23]. Computational cost is crucial in this application so that instead of a detailed/truth model, simplified models are used. Vehicle 
dynamics models in this application are used to provide feasible paths between particular points. Other algorithms use these paths to generate the planned path [21].

Simplified, longitudinal vehicle dynamics models are used in the case of powertrain analysis and optimization [24], [25]. The main reason for this simplification is that several details of vehicle dynamics are not significant in this application. The main focus is on the powertrain modeling itself, with the utilization of a simplified, longitudinal vehicle dynamics model.

The simplified-detailed contrast of vehicle models can be observed even in the field of structural vehicle models. In reference [31] simplified, multibody-based crashworthiness vehicle models are identified based on detailed finite element (FE) structural models of the vehicle. This application is related to vehicle structural models instead of vehicle dynamics models. In vehicle dynamics models, the detailed ones are based on the multibody approaches.

\subsection{Application of detailed vehicle models}

The main property of detailed/truth vehicle models [26], [27] is that the vehicle body has all degrees of freedom, there are no constraints applied to the body. Contact modeling is crucial in this kind of models, the intersection must be considered between the tire and the road because of the lack of constraints of the vehicle body. Detailed vehicle models usually consider vehicles on rough surfaces with the modeling of their suspensions. These types of vehicle models are usually multibody systems. Other detailed phenomena like combined slip scenarios and relaxation length properties of tires have to be also considered, along with the air resistance and powertrain modeling. There are software environments (e.g. IPG CarMaker and veDYNA) providing detailed physical plants of vehicles, with interfaces to other software (e.g. MATLAB/Simulink) where custom algorithms can be designed and run parallel with the physical plant of the car.

\subsubsection{Control/estimation algorithm evaluation}

Control/estimation algorithms in their development phase can be evaluated based on either experimental (field) data or simulated data. Only the final validation of the algorithms has to be performed based on experiments. This can accelerate the development time of such algorithms, and the robustness of these algorithms also can be checked more thoroughly with simulations. Model-inLoop (MIL), Software-in-Loop (SIL) and Hardware-in-Loop (HIL) methods are used [28], [29] first to develop the algorithms, then the hardware implementation is also well-analyzed, developed and validated.

\subsubsection{Advanced sensor modeling, computer graphics}

There are sensors which relatively easy to model: sensors in connection with motion and position. These kinds of sensors are related to the physical plant of the vehicle. However, there are sensors (cameras, radars, and lidars) which detect the environment of the car. For the modeling purpose of these sensors, the environment of the vehicle also has to be modeled, which can be achieved by the means of computer graphics. Full autonomous driving systems can be virtually prototyped based on 
the detailed physical plants, and this kind of advanced sensor models. Software environments like IPG CarMaker and veDYNA provide tools to virtually validate autonomous driving systems.

\section{Modeling considerations of vehicle dynamics models}

\subsection{Longitudinal vehicle models}

In Simple Vehicle Model (Figure 1) the vehicle is considered as one rigid body which moves along an ideally even and horizontal road. At each axle the forces in the wheel contact points are combined in one normal and one longitudinal force [34]-[36].

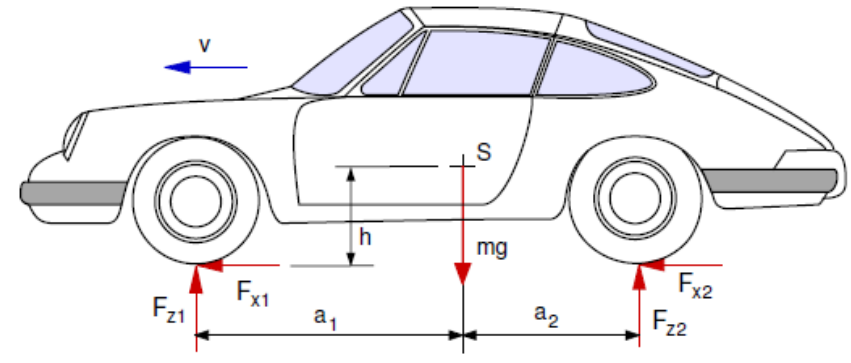

Figure 1. Forces acting on the vehicle body in the Simple Vehicle Model [34]

By neglected aerodynamic forces (drag, positive and negative lift), the equations of motions in the $\mathrm{x}$-, z-plane are presented in the following mathematical forms

$$
\begin{array}{r}
m \dot{v}=F_{x 1}+F_{x 2} \\
0=F_{z 1}+F_{z 2}-m g \\
0=F_{z 1} a_{1}-F_{z 2} a_{2}+\left(F_{x 1}+F_{x 2}\right) h
\end{array}
$$

where $v$ derivate indicates the vehicle acceleration, $m$ is the mass of the vehicle, $a_{1}+a_{2}$ is the wheel base, and $h$ is the height of the center of gravity.

There are static parts of the equations which can be described as

$$
F_{z 1}^{s t}=m g \frac{a_{2}}{a_{1}+a_{2}}, \quad F_{z 2}^{s t}=m g \frac{a_{1}}{a_{1}+a_{2}}
$$

The height of the center of gravity only influences the dynamic part of the axle loads [34]. Moreover, the statics forces there are dynamical forces which emerge when the vehicle is moving in the analysis.

$$
F_{z 1}^{d y n}=-m g \frac{h}{a_{1}+a_{2}} \frac{\dot{v}}{g}, \quad F_{z 2}^{d y n}=+m g \frac{h}{a_{1}+a_{2}} \frac{\dot{v}}{g}
$$

When accelerating $\dot{v}>0$, the front axle is relieved. The rear axle is relieved in case of the situation when decelerating $\dot{v}<0$.

Next, the influence of grade is presented in the paper, Figure 2 shows the acting forces on the vehicle in this case [37]. 


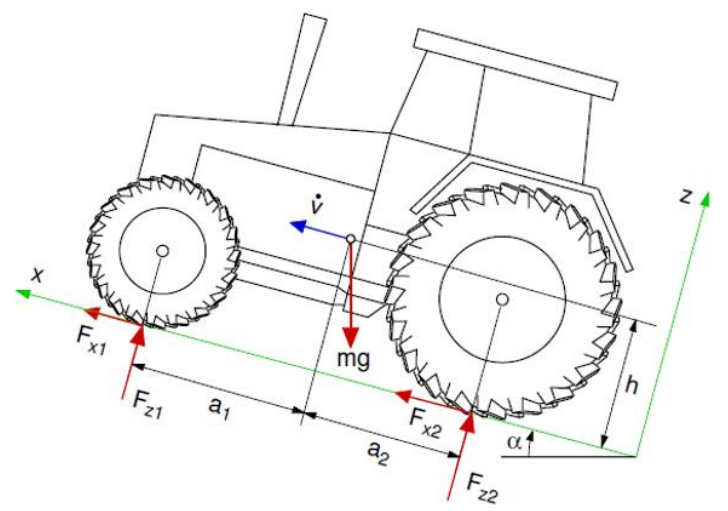

Figure 2. Influences of the grade on the vehicle considering the forces [34]

As for aerodynamic forces, the shape of most vehicles produce aerodynamic forces and torques as Figure 3 presents. There are other cases when specific wings are mounted on the vehicle in order to produce excessive forces even at higher speed of the vehicle [35], [38]. Effect of these aerodynamic forces and torques can be represented by a resistant force applied at the center of gravity and down forces acting at the front and rear axle by the following mathematical equations

$$
\begin{aligned}
& F_{z 1}=m g \cos \alpha \frac{a_{2}-h \tan \alpha}{a_{1}+a_{2}}-\frac{h}{a_{1}+a_{2}} m \dot{v} \\
& F_{z 2}=m g \cos \alpha \frac{a_{1}-h \tan \alpha}{a_{1}+a_{2}}+\frac{h}{a_{1}+a_{2}} m \dot{v}
\end{aligned}
$$

where the dynamic parts remain unchanged, $\alpha$ denotes the grade angle. Now the static parts also depend on the grade angle and the height of the center of gravity.

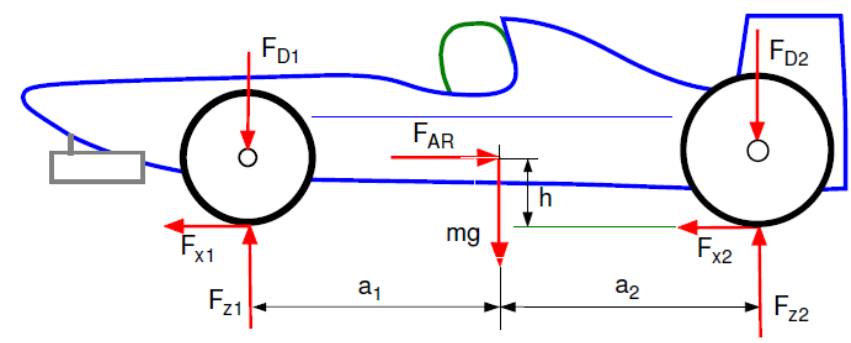

Figure 3. Forces on the vehicle body with the aerodynamic effect [34]

If we assume a positive driving speed, $v>0$, the equations of motion will read as

$$
\begin{array}{r}
m \dot{v}=F_{x 1}+F_{x 1}-F_{A R} \\
0=F_{z 1}-F_{D 1}+F_{z 2}-F_{D 2}-m g \\
0=\left(F_{z 1}-F_{D 1}\right) a_{1}-\left(F_{z 2}-F_{D 2}\right) a_{2}+\left(F_{x 1}+F_{x 2}\right) h
\end{array}
$$

where $F_{A R}$ and $F_{D 1}, F_{D 2}$ describe the air resistance and the down forces. For the dynamic axle loads we get

$$
F_{z 1}=F_{D 1}+m g \frac{a_{2}}{a_{1}+a_{2}}-\frac{h}{a_{1}+a_{2}}\left(m \dot{v}+F_{A R}\right)
$$




$$
F_{z 2}=F_{D 2}+m g \frac{a_{1}}{a_{1}+a_{2}}+\frac{h}{a_{1}+a_{2}}\left(m \dot{v}+F_{A R}\right)
$$

As for the maximum acceleration analysis and if aerodynamic forces are the part of the dynamical model, the maximum acceleration and deceleration on a horizontal road can be expressed by the next equations

$-\mu\left(1+\frac{F_{D 1}}{m g}+\frac{F_{D 2}}{m g}\right)-\frac{F_{A R}}{m g} \leq \frac{\dot{v}}{g} \leq \mu\left(1+\frac{F_{D 1}}{m g}+\frac{F_{D 2}}{m g}\right)-\frac{F_{A R}}{m g}$

In case of linear motion the motion is described by the equation

$$
m \dot{v}_{R W D}=\mu m g\left[\frac{a_{1}}{a_{1}+a_{2}}+\frac{h}{a_{1}+a_{2}} \frac{\dot{v}_{R W D}}{g}\right]
$$

where the subscript RWD indicates the rear wheel drive. Maximum acceleration for a rear wheel driven vehicle can be calculated by

$$
\frac{\dot{v}_{R W D}}{g}=\frac{\mu}{1-\mu \frac{h}{a_{1}+a_{2}}} \frac{a_{1}}{a_{1}+a_{2}}
$$

By setting $F_{x 1}=\mu F_{z 1}$ and $F_{z 1}=0$, the maximum acceleration for a front wheel driven vehicle can be calculated in a similar way the acceleration for front drive vehicles can be calculated as

$$
\frac{\dot{v}_{F W D}}{g}=\frac{\mu}{1-\mu \frac{h}{a_{1}+a_{2}}} \frac{a_{1}}{a_{1}+a_{2}}
$$

where the subscript $F W D$ denotes front wheel drive.

Tire is the main component of a vehicle interacting with the road. The performance of a vehicle is mainly influenced by the characteristics of its tires. Tires affect a vehicle handling, traction, ride comfort, and fuel consumption. To understand its importance, it is enough to remember that a vehicle can maneuver only by longitudinal, vertical, and lateral force systems generated under the tires [39].

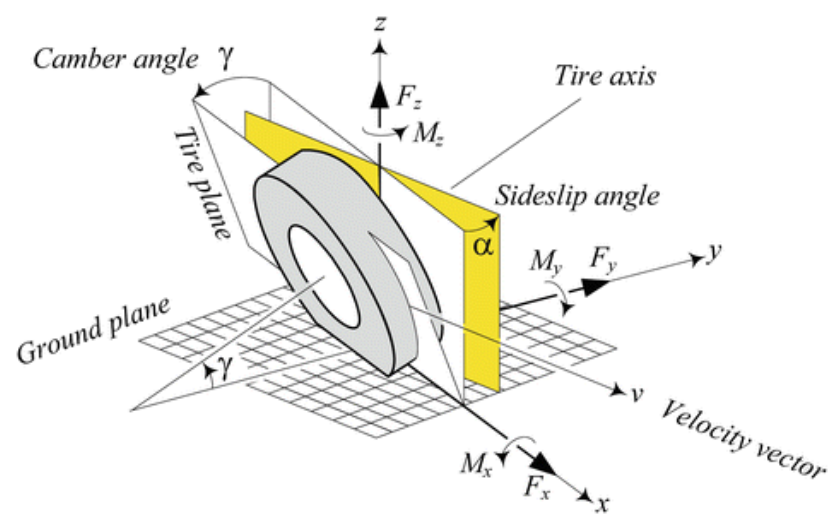

Figure 4. Forces acting on the vehicle tyre [40]

A vehicle is made to move or change its direction in a specific way by forces acting through the tires. The types of the tires play important role on the behavior of the vehicle. The braking distance is 
dependent on a number of factors including vehicle speed. At a constant rate of deceleration, braking distance increases quadratically relative to speed [39], [41].

As for the vehicle load, extra weight makes braking distances longer. Road conditions are important, wet roads offer less adhesion between road surface and tires and therefore result in longer braking distances. In case of tire condition, insufficient tread depth increases braking distances, particularly on wet road surfaces.

The circumferential force is produced by power transmission or braking. It acts on the road surface as a linear force in line with the longitudinal axis of the vehicle and enables the driver to increase the speed of the vehicle using the accelerator or slow it down with the brakes.

The vertical force acting downwards between the tire and road surface is called the vertical tire force or normal force. It acts on the tires [34], [40] at all times regardless of the state of motion of the vehicle, including, therefore, when the vehicle is stationary.

The vertical force is determined by the proportion of the combined weight of vehicle and payload that is acting on the individual wheel concerned [42]. It also depends on the degree of upward or downward gradient of the road that the vehicle is standing on.

Other forces acting on the vehicle can increase or decrease the vertical force. When cornering, the force is reduced on the inner wheels and increased on the outer wheels. The suspension of the vehicle play significant role in the road-tire interactions [43].

The analysis of other forces from other directions on the vehicle body are the part of the next sections.

\subsection{Lateral vehicle models}

The so-called lateral vehicle models are often used in the vehicle dynamics. The aim of these kinds of models is to analyse the behaviour, manoeuvrability and stability of the vehicle during cornering. For this purpose many kinds of models can be found in the literature [44]-[46]. The type of the applied model depends on the speed of vehicle. For moderate speed cornering cases a simple kinematical model can be used to analyse the cornering circumstances of the vehicle. The most commonly applied model for this purpose - the so-called Ackermann steering geometry - is shown in Figure 5. Here, all wheels rotation axes intersect in one point. For low vehicle speed, the Ackermann steering geometry gives best manoeuvrability and lowest tyre wear. 


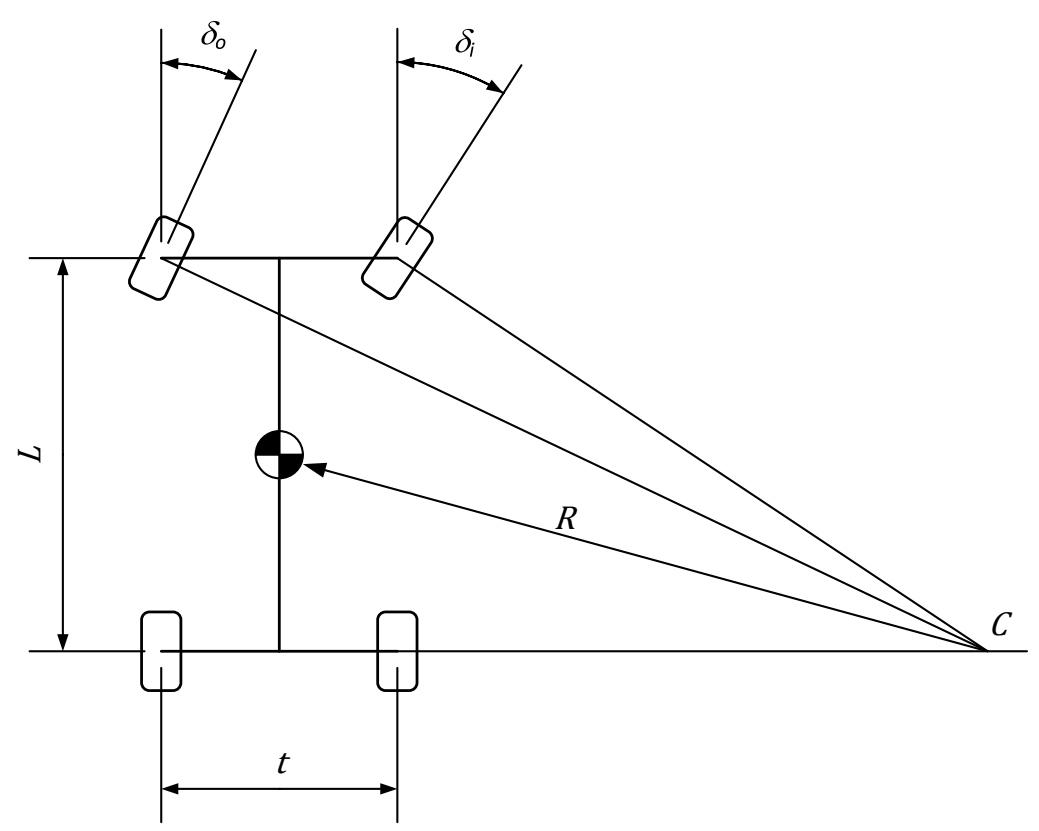

Figure 5. Ackermann steering geometry

The relation between the wheels steering angles is presented in the following equation.

$$
\frac{1}{\tan \delta_{o}}=\frac{1}{\tan \delta_{i}}+\frac{t}{L}
$$

In the case of high speed cornering, the lateral dynamic effects, inertial forces and the lateral slip of wheels cannot be neglected any more. Here, the so-called one-track model (a.k.a. bicycle model) is a common choice for modelling the lateral dynamics of vehicle, see Figure 6. In this model the effects of both tyres on the axle is combined into one virtual tyre. The lateral tyre slips of both axes as well as the instantaneous cornering centre of the vehicle are presented in Figure 6.

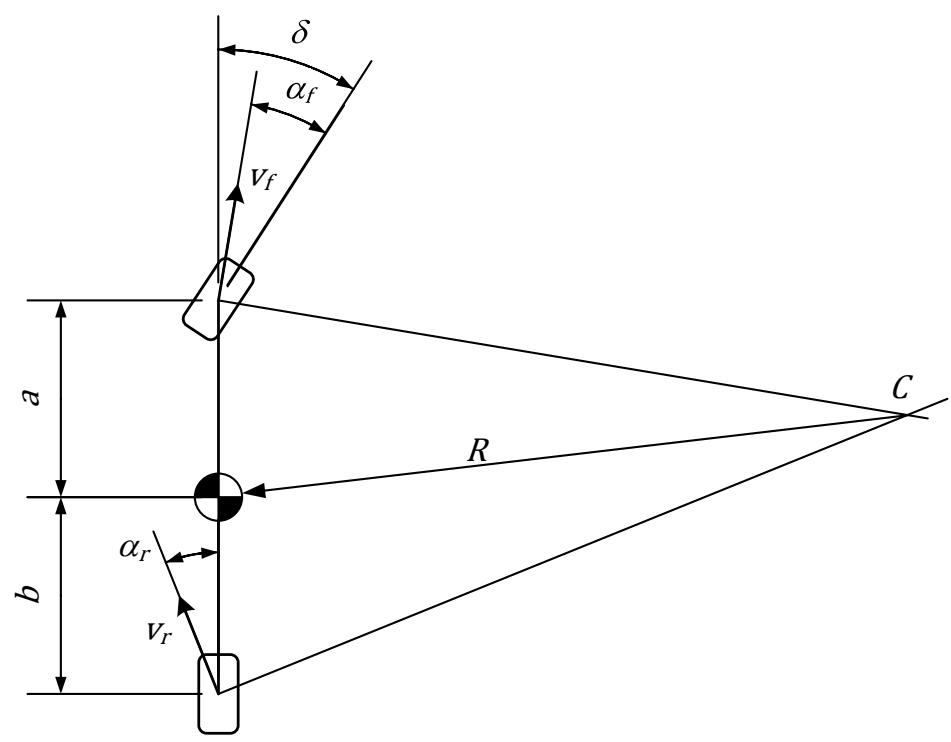

Figure 6. Bicycle model 


\subsection{Vertical vehicle models}

The purpose of the vertical dynamic models is to investigate the motion, vibrations of the vehicles in the vertical direction [47], [48]. By using these models, the suspension behaviour, comfort of the vehicle can be analysed as well as the design and fatigue analysis of the suspension components can be performed [49]. The design of active and semi-active suspension control systems is also based on these types of dynamic models [50]. The simplest vertical dynamic model for vehicles - the so-called quarter-car model - is presented in Figure 7. In this model the vertical movement of the vehicle is reduced to one suspension system. The sprung- and unsprung masses as well as the stiffness and damping effects of the tyre and the suspension system are the main parameters of the presented model.

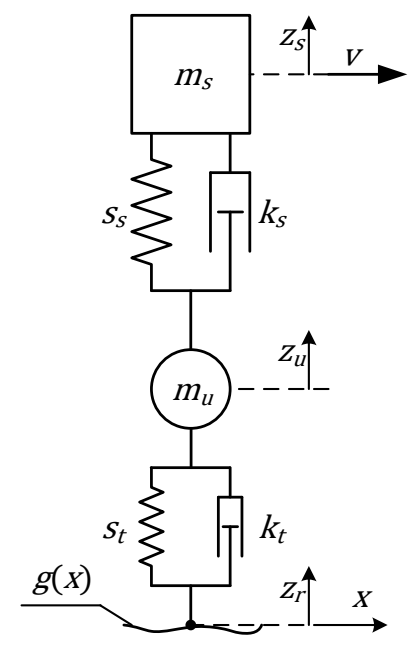

Figure 7. Quarter-car model

The governing differential equations of motion for the quarter-car model are presented in the following equations.

$$
\begin{gathered}
m_{s} \ddot{z}_{s}+k_{s} \dot{z}_{s}-k_{s} \dot{z}_{u}+s_{s} z_{s}-s_{s} z_{u}=0 \\
m_{u} \ddot{z}_{u}-k_{s} \dot{z}_{s}+\left(k_{s}+k_{t}\right) \dot{z}_{u}-s_{s} z_{s}+\left(s_{s}+s_{t}\right) z_{u}=k_{t} \dot{z}_{r}+s_{t} z_{r}
\end{gathered}
$$

A more complex vertical dynamic model - the so-called half-car model - is presented in Figure 8. This is still a planar model, however, in this model the pitch motion of the vehicle is also taken into account. The governing differential equations of motion for the half-car model ([51], [52]) are presented in Equations (20-23). 


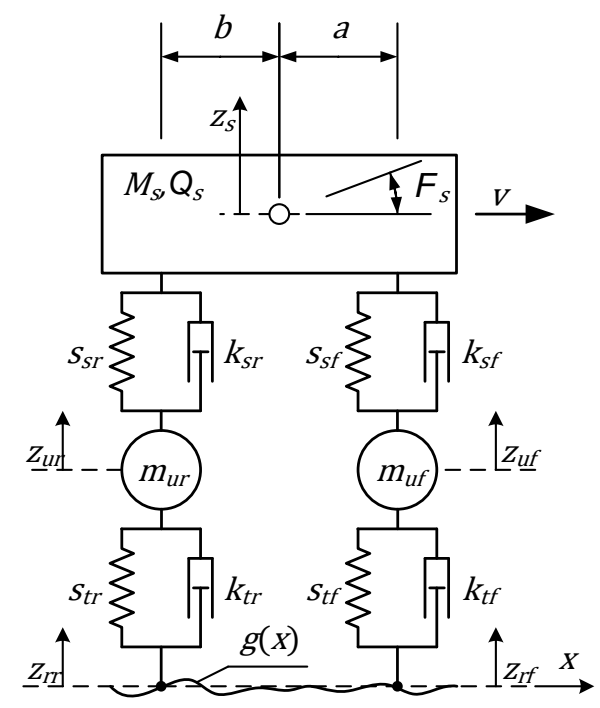

Figure 8. Half-car model

$$
\begin{gathered}
m_{u f} \ddot{z}_{u f}+\left(k_{t f}+k_{s f}\right) \dot{z}_{u f}-k_{s f} \dot{z}_{s}+a k_{s f} \dot{\phi}_{s}+\left(s_{t f}+s_{s f}\right) z_{u f}-s_{s f} z_{s}+a s_{s f} \phi_{s}=k_{t f} \dot{z}_{r f}+s_{t f} z_{r f} \\
m_{u r} \ddot{z}_{u r}+\left(k_{t r}+k_{s r}\right) \dot{z}_{u r}-k_{s r} \dot{z}_{s}-b k_{s r} \dot{\phi}_{s}+\left(s_{t r}+s_{s r}\right) z_{u r}-s_{s r} z_{s}-b s_{s r} \phi_{s}=k_{t r} \dot{z}_{r r}+s_{t r} z_{r r} \\
M_{s} \ddot{z}_{s}-k_{s f} \dot{z}_{u f}-k_{s r} \dot{z}_{u r}+\left(k_{s f}+k_{s r}\right) \dot{z}_{s}+\left(-a k_{s f}+b k_{s r}\right) \dot{\phi}_{s}- \\
-s_{s f} z_{u f}-s_{s r} z_{u r}+\left(s_{s f}+s_{s r}\right) z_{s}+\left(-a s_{s f}+b s_{s r}\right) \phi_{s}=0 \\
\Theta_{s} \ddot{\phi}_{s}+a k_{s f} \dot{z}_{u f}-b k_{s r} \dot{z}_{u r}+\left(-a k_{s f}+b k_{s r}\right) \dot{z}_{s}+\left(a^{2} k_{s f}+b^{2} k_{s r}\right) \dot{\phi}_{s}+ \\
+a s_{s f} z_{u f}-b s_{s r} z_{u r}+\left(-a s_{s f}+b s_{s r}\right) z_{s}+\left(a^{2} s_{s f}+b^{2} s_{s r}\right) \phi_{s}=0
\end{gathered}
$$

\section{Conclusions}

Vehicle dynamics models in various application fields have been analyzed and classified. It has been shown that the utilized model is highly dependent on the application. Several simplified models have been presented in detail.

\section{Acknowledgments}

The research was financed by the Thematic Excellence Programme of the Ministry for Innovation and Technology in Hungary (ED_18-1-2019-0028), within the framework of the (Automotive Industry) thematic programme of the University of Debrecen.

\section{References}

[1] M. L. McIntyre, T. J. Ghotikar, A. Vahidi, X. Song, and D. M. Dawson, "A two-stage Lyapunov-based estimator for estimation of vehicle mass and road grade," IEEE Trans. Veh. Technol., vol. 58, no. 7, pp. 3177-3185, 2009.

[2] H. Ohnishi, J. Ishii, M. Kayano, and H. Katayama, "Study on road slope estimation for automatic transmission control," JSAE Rev., vol. 21, no. 2, pp. 235-240, 2000. 
[3] K. Enisz, I. Szalay, G. Kohlrusz, and D. Fodor, "Tyre-road friction coefficient estimation based on the discrete-time extended Kalman filter," Proc. Inst. Mech. Eng. Part D J. Automob. Eng., vol. 229, no. 9, pp. 1158-1168, 2015.

[4] A. Nishio, K. Tozu, H. Yamaguchi, K. Asano, and Y. Amano, "Development of vehicle stability control system based on vehicle sideslip angle estimation," SAE Tech. Pap., no. 724, 2001.

[5] S. Fergani, L. Menhour, O. Sename, L. Dugard, and B. D'Andrea Novel, "Study and comparison of non linear and LPV control approaches for vehicle stability control," 21st Mediterr. Conf. Control Autom. MED 2013 - Conf. Proc., no. September 2015, pp. 303-310, 2013.

[6] G. Baffet, A. Charara, and D. Lechner, "Estimation of vehicle sideslip, tire force and wheel cornering stiffness," Control Eng. Pract., vol. 17, no. 11, pp. 1255-1264, 2009.

[7] N. Hamzah, Y. M. Sam, H. Selamat, M. K. Aripin, and M. F. Ismail, "Yaw stability improvement for four-wheel active steering vehicle using sliding mode control," Proc. - 2012 IEEE 8th Int. Colloq. Signal Process. Its Appl. CSPA 2012, pp. 127-132, 2012.

[8] S. Ikenaga, F. L. Lewis, J. Campos, and L. Davis, "Active suspension control of ground vehicle based on a full-vehicle model," Proc. Am. Control Conf., vol. 6, no. June, pp. 4019-4024, 2000.

[9] G. Koch, T. Kloiber, E. Pellegrini, and B. Lohmann, "A nonlinear estimator concept for active vehicle suspension control," Proc. 2010 Am. Control Conf. ACC 2010, no. 2, pp. 4576-4581, 2010.

[10] L. R. Ray, "Nonlinear Tire Force Estimation and Road Friction Identification: Simulation and Experiments," Automatica, vol. 33, no. 10, pp. 1819-1833, 1997.

[11] N. K. M’Sirdi, A. Rabhi, N. Zbiri, and Y. Delanne, "Vehicle-road interaction modelling for estimation of contact forces," Veh. Syst. Dyn., vol. 43, no. SUPPL., pp. 403-411, 2005.

[12] V. Cerone, M. Milanese, and D. Regruto, "Combined Automatic Lane-Keeping and Driver's Steering Through a 2-DOF Control Strategy," IEEE Trans. Control Syst. Technol., vol. 17, no. 1, pp. 135-142, 2008.

[13] L. S. Miller, "Predictive control of autonomous ground vehicles with obstacle avoidance on slippery roads," Arch. Phys. Med. Rehabil., vol. 70, no. 12, pp. 866-867, 1989.

[14] Y. Yoon, J. Shin, H. J. Kim, Y. Park, and S. Sastry, "Model-predictive active steering and obstacle avoidance for autonomous ground vehicles," Control Eng. Pract., vol. 17, no. 7, pp. 741-750, 2009.

[15] P. Taylor, a J. C. Schmeitz, I. J. M. Besselink, and S. T. H. Jansen, "Road Friction Coefficient Estimation For Vehicle Path Prediction,” Veh. Syst. Dyn., vol. 53, no. March 2014, pp. 37-41, 2012.

[16] H. Sado and S. Sakai Yoichi, "Road Condition Estimation for Traction Control," ISIE'99. Proc. IEEE Int. Symp. Ind. Electron., vol. 2, pp. 973-978, 1999.

[17] D. Ben Hassen, M. Miladi, M. S. Abbes, S. C. Baslamisli, F. Chaari, and M. Haddar, "Road profile estimation using the dynamic responses of the full vehicle model," Appl. Acoust., vol. 147, no. October, pp. 87-99, 2019.

[18] M. Doumiati, A. Victorino, A. Charara, and D. Lechner, "Estimation of road profile for vehicle dynamics motion: Experimental validation," Proc. Am. Control Conf., pp. 5237-5242, 2011.

[19] K. Jo, K. Chu, and M. Sunwoo, "Interacting multiple model filter-based sensor fusion of GPS with in-vehicle sensors for real-time vehicle positioning," IEEE Trans. Intell. Transp. Syst., vol. 13, no. 1, pp. 329-343, 2012.

[20] P. J. T. H. Venhovens and K. Naab, "Vehicle Dynamics Estimation Using Kalman Filters," Veh. Syst. Dyn. Int. J. Veh. Mech. Mobil., no. October 2014, pp. 37-41, 2010. 
[21] M. Likhachev and D. Ferguson, "Planning long dynamically feasible maneuvers for autonomous vehicles," Int. J. Rob. Res., vol. 28, no. 8, pp. 933-945, 2009.

[22] R. Pepy, A. Lambert, and H. Mounier, "Path Planning using a Dynamic Vehicle Model," 2006 2nd Int. Conf. Inf. Commun. Technol., vol. 1, no. 1, p. 1, 2006.

[23] J. Kong, M. Pfeiffer, G. Schildbach, and F. Borrelli, "Kinematic and dynamic vehicle models for autonomous driving control design," IEEE Intell. Veh. Symp. Proc., vol. 2015-Augus, no. Iv, pp. 1094-1099, 2015.

[24] L. V. Pérez, G. R. Bossio, D. Moitre, and G. O. García, "Optimization of power management in an hybrid electric vehicle using dynamic programming," Math. Comput. Simul., vol. 73, no. 1-4 SPEC. ISS., pp. 244-254, 2006.

[25] S. Mahapatra, T. Egel, R. Hassan, R. Shenoy, and M. Carone, "Model-based design for hybrid electric vehicle systems," SAE Tech. Pap., vol. 2008, no. 724, 2008.

[26] M. W. Sayers and D. Han, "A generic multibody vehicle model for simulating handling and braking," Veh. Syst. Dyn., vol. 25, no. SUPPL., pp. 599-613, 1996.

[27] A. Szántó and S. Hajdu, "Vehicle Modelling and Simulation in Simulink," Int. J. Eng. Manag. Sci., vol. 4, no. 1, pp. 260-265, 2019.

[28] K. N. Kota and B. Sivanandham, "Integrated Model-In-Loop (MiL) Simulation Approach to Validate Active Roll Control System," SAE Tech. Pap., vol. 2017-March, no. March, 2017.

[29] D. Fodor and K. Enisz, "Vehicle dynamics based ABS ECU verification on real-time hardware-inthe-loop simulator," 16th Int. Power Electron. Motion Control Conf. Expo. PEMC 2014, pp. 1247$1251,2014$.

[30] L. Sousa, P. Veríssimo, and J. Ambrósio, "Development of generic multibody road vehicle models for crashworthiness," Multibody Syst. Dyn., vol. 19, no. 1-2, pp. 133-158, 2008.

[31] A. V. Pesterev, L. A. Bergman, and C. A. Tan, "A novel approach to the calculation of potholeinduced contact forces in MDOF vehicle models," J. Sound Vib., vol. 275, no. 1-2, pp. 127-149, 2004.

[32] G. Rill, “TMeasy-A Handling Tire Model based on a three-dimensional slip approach,” ... XXIII Int. Symp. ..., pp. 1-10, 2013.

[33] J. P. Maurice and H. B. Pacejka, "Relaxation length behaviour of tyres," Veh. Syst. Dyn., vol. 27, no. Suppl, pp. 37-41, 1997.

[34] G. Rill, "Fundamental Vehicle Dynamics," no. August, 2011, pp. 1-12.

[35] M. Hirz, "Automotive Engineering Focus : Basics of longitudinal vehicle dynamics," no. July, p. $26,2015$.

[36] D. Hernandez-Alcantara, L. Amezquita-Brooks, R. Morales-Menendez, O. Sename, and L. Dugard, "The cross-coupling of lateral-longitudinal vehicle dynamics: Towards decentralized FaultTolerant Control Schemes," Mechatronics, vol. 50, pp. 377-393, 2018.

[37] Z. Yin, Q. Dai, H. Guo, H. Chen, and L. Chao, "Estimation Road Slope and Longitudinal Velocity for Four-wheel Drive Vehicle," IFAC-PapersOnLine, vol. 51, no. 31, pp. 572-577, 2018.

[38] B. Janarthanan, C. Padmanabhan, and C. Sujatha, "Longitudinal dynamics of a tracked vehicle: Simulation and experiment," J. Terramechanics, vol. 49, no. 2, pp. 63-72, 2012.

[39] E. Joa, K. Yi, and Y. Hyun, "Estimation of the tire slip angle under various road conditions without tire-road information for vehicle stability control," Control Eng. Pract., vol. 86, pp. 129143, May 2019. 
[40] R. N. Jazar, Vehicle Dynamics. Theory and Applications. .

[41] J. Hu, S. Rakheja, and Y. Zhang, "Tire-Road Friction Coefficient Estimation under Constant Vehicle Speed Control," IFAC-PapersOnLine, vol. 52, no. 8, pp. 283-288, 2019.

[42] I. Blekhman and E. Kremer, "Vertical-longitudinal dynamics of vehicle on road with unevenness," Procedia Eng., vol. 199, pp. 3278-3283, 2017.

[43] A. G. Mohite and A. C. Mitra, "Development of Linear and Non-linear Vehicle Suspension Model," Mater. Today Proc., vol. 5, no. 2, pp. 4317-4326, Jan. 2018.

[44] G. Šušteršič, M. Ambrož, and I. Prebil, "Application of Rigid Multi-Body System Modelling to Determination of Passenger-Car and Trailer Combination Lateral Stability," in SCIENTIFIC PROCEEDINGS XIX INTERNATIONAL SCIENTIFIC-TECHNICAL CONFERENCE "trans \& MOTAUTO '11', 2011, vol. 3, pp. 94-97.

[45] N. El Youssfi, M. Oudghiri, and R. El Bachtiri, "Vehicle lateral dynamics estimation using unknown input observer," Procedia Comput. Sci., vol. 148, pp. 502-511, 2019.

[46] M. N. Özdemir, V. Kılıç, and Y. S. Ünlüsoy, "A new contact \&amp; slip model for tracked vehicle transient dynamics on hard ground," J. Terramechanics, vol. 73, pp. 3-23, Oct. 2017.

[47] G. Georgiou, A. Badarlis, and S. Natsiavas, "Modelling and ride dynamics of a flexible multi-body model of an urban bus," Proc. Inst. Mech. Eng. Part KJ. Multi-body Dyn., vol. 222, no. 2, pp. 143154, Jun. 2008.

[48] J. D. Setiawan, M. Safarudin, and A. Singh, "Modeling, simulation and validation of 14 DOF full vehicle model," in International Conference on Instrumentation, Communication, Information Technology, and Biomedical Engineering 2009, 2009, no. August 2015, pp. 1-6.

[49] M. Haiba, D. C. Barton, P. C. Brooks, and M. C. Levesley, "Using a quarter-vehicle multi-body model to estimate the service loads of a suspension arm for durability calculations," Proc. Inst. Mech. Eng. Part KJ. Multi-body Dyn., vol. 217, no. 2, pp. 121-133, Jun. 2003.

[50] Z. Kong, D. Pi, X. Wang, H. Wang, and S. Chen, "Design and Evaluation of a Hierarchical Control Algorithm for an Electric Active Stabilizer Bar System," Strojniški Vestn. - J. Mech. Eng., vol. 62, no. 10, pp. 565-576, Oct. 2016.

[51] M. Mirzaei and R. Hassannejad, "Application of genetic algorithms to optimum design of elastodamping elements of a half-car model under random road excitations," Proc. Inst. Mech. Eng. Part KJ. Multi-body Dyn., vol. 221, no. 4, pp. 515-526, Dec. 2007.

[52] H. El-taweel, M. M. Abd elhafiz, and H. Metered, "Optimal lumped parameters estimation of vehicle passive suspension system using genetic algorithm," J. Adv. Veh. Eng., vol. 5, no. 1, pp. 34-44, 2018. 